\title{
On Euclidean diagrams and geometrical knowledge ${ }^{8}$
}

\author{
(Sobre los diagramas euclidianos y el conocimiento geométrico)
}

\author{
Tamires Dal Magro*1, Manuel J. García-PÉReZ ${ }^{2}$ \\ ${ }^{1}$ Universidade Estadual de Campinas \\ ${ }^{2}$ Universidad de Sevilla
}

\begin{abstract}
We argue against the claim that the employment of diagrams in Euclidean geometry gives rise to gaps in the proofs. First, we argue that it is a mistake to evaluate its merits through the lenses of Hilbert's formal reconstruction. Second, we elucidate the abilities employed in diagram-based inferences in the Elements and show that diagrams are mathematically reputable tools. Finally, we complement our analysis with a review of recent experimental results purporting to show that, not only is the Euclidean diagram-based practice strictly regimented, it is rooted in cognitive abilities that are universally shared.
\end{abstract}

KEYWORDS: mathematical practice; Euclidean geometry; diagrammatic reasoning; cognitive abilities.

RESUMEN: Argumentamos en contra de la afirmación de que el uso de diagramas en la geometría euclidiana da lugar a vacios o lagunas en las pruebas. En primer lugar, mostramos que es un error evaluar sus méritos a través de las lentes de la reconstrucción formal de Hilbert. En segundo lugar, esclarecemos las habilidades empleadas en las inferencias basadas en los diagramas en los Elementos, y mostramos que los diagramas son herramientas matemáticas respetables. Finalmente, complementamos nuestro análisis con una revisión de resultados experimentales recientes que pretenden mostrar que la práctica diagramática euclidiana no solo está estrictamente regimentada, sino que también está enraizada en ciertas habilidades cognitivas universalmente compartidas.

PALABRAS CLAVE: práctica matemática; geometría euclidiana; razonamiento diagramático; habilidades cognitivas.

\& This research was supported by Fundação de Amparo à Pesquisa do Estado de São Paulo (FAPESP, grant 2016/20480-5 and 2014/23191-9). We wish to thank José Ferreirós, María de Paz, Matheus Valente, Rogerio Passos Severo, Abel Lassalle Casanave, Emiliano Boccardi and Marco Ruffino for insightful discussions and suggestions on earlier drafts of this paper, as well as the referees for their helpful comments.

* Correspondence to: Tamires Dal Magro. Instituto de Filosofia e Ciências Humanas. Universidade Estadual de Campinas (UNICAMP). R/ Cora Coralina, 100, 13083-896, Cidade Universitária Zeferino Vaz, Barão Geraldo, Campinas, São Paulo, Brasi - tamiresdma@gmail.comhttps://orcid.org/0000-0001-7423-9223

How to cite: Dal Magro, Tamires; García-Pérez, Manuel J. (2019). «On Euclidean diagrams and geometrical knowledge»; Theoria. An International Journal for Theory, History and Foundations of Science, 34(2), 255-276. (https://doi.org/10.1387/theoria.20026).

Received: 27 June, 2018; Final version: 22 March, 2019.

ISSN 0495-4548 - elSSN 2171-679X / (C) 2019 UPV/EHU 


\section{Introduction}

A widely held view in the philosophy of mathematics attributes to diagrams a merely heuristic or illustrative-and thus dispensable - role in mathematical demonstrations. This tradition conceives of demonstrations as syntactic objects made up of finite and inspectable arrangements of sentences (Tennant 1986). Thus, demonstrations that make essential use of diagrams-i.e. that make use of information provided by drawings-came to be viewed as non-rigorous and of no interest to philosophy (Borwein 2008); such was the case with the demonstrations found in Euclid's Elements. Information extracted from diagrams was then considered to give rise to argument gaps.

This view can be found in the work of many nineteenth-century authors, such as Pasch, who affirms that "the theorem is only truly demonstrated if the proof is completely independent of the figure" (Pasch 1882/1926, 43 apud Mancosu 2005, 14). Pasch is well known for having pioneered geometries without diagrams. Similarly, Hilbert claims in his lectures on the foundations of geometry that the use of figures can easily be misleading and, for this reason, a theorem is only proved when the proof is completely independent of the figure. ${ }^{1}$ Even earlier, Dedekind had expressed dissatisfaction about the appeal to geometric intuitions (understood as a form of visual perception) in basic infinitesimal analysis and claimed that the concepts employed therein were vague and obscure (Dedekind 1963). In the same vein, Russell (1949 [1901]) argued that diagrams have no epistemic role in geometry: "formerly, it was held by philosophers and mathematicians alike that the proofs in Geometry depended on the figure; nowadays, this is known to be false. In the best books there are no figures at all. The reasoning proceeds by the strict rules of formal logic from a set of axioms laid down to begin with" (93). The author explicitly admitted not trusting Euclidean geometrical reasoning's validity and went as far as claiming that there was no correct mathematical reasoning before the eighteenth century (Russell 1919, 145).

Legris (2012) remarks that the rejection of the epistemic relevance of diagrams has its roots in the development of symbolic logic and the discussions about the foundations of mathematics that took place in the late nineteenth and early twentieth centuries. ${ }^{2}$ That rejection, supported by authors like Russell, Hilbert and Tarski, led to a conception of mathematical demonstration modelled on the proofs of symbolic logic: a sequence of sentences each one of which is either an axiom or a sentence that follows from earlier sentences in the sequence via inference rules. Since inference rules are rules of syntactic transformation, that conception naturally leads one to the view that demonstrations are purely syntactical objects. The reconstruction of a demonstration in those terms was supposed to exhibit its underlying logical form, and allow one to see if it indeed contains a finite sequence of formulas (a supposedly sine qua non condition on proper demonstrations).

Given this definition of proof-pervasive in logic textbooks of the time, such as Carnap (1939), and Church (1956) — the standard views on mathematical rigor and knowledge were tied to the possibility of a formal reconstruction of demonstrations. As Ferreirós (2016) summarizes: "Clarifying what proof and rigor meant was the task of logical analysis, which led to

See Hilbert's 1894 lecture in Hallett \& Majer (2004).

2 See Giaquinto (2007) for concrete examples of mathematical cases where diagrams seemed to lead to fallacious conclusions and an explanation of why these cases do not constitute a good induction base for the claim that diagrams can often lead to fallacious conclusions. 
the twentieth-century identification of 'rigor' with respect for the rules of a logical calculus, and 'proof with certain sequences of strings of symbols in formal systems" (24).

However, as the author points out, defining those notions in those terms brings about multiple tensions when we confront actual mathematical practice. Firstly, mathematicians from the past did not share that conception of rigor (indeed, Euclid's Elements was considered for many centuries the paradigm of mathematical rigor). ${ }^{3}$ Secondly, even for those acquainted with current mathematical practice, it is clear that there are more elements involved in a proof than propositions sequentially following from one another. In relation to that, Ferreirós remarks that "the interest of practicing mathematicians in multiple proofs of one and the same result, for instance, is evidence that there is more methodology behind the conduct of proofs than a narrow-minded logical analysis reveals" $(2016,24-25) .{ }^{4} \mathrm{Fi}$ nally, the claim that diagrams are not rigorous mathematical tools starkly conflicts with the fact that ancient mathematical practices, such as the Euclidean one, were greatly successful and free of errors.

These are some reasons why Ferreirós (2016) suggests that understanding how we attain mathematical knowledge crucially depends on an analysis of mathematical practices, defined as "what the community of mathematicians does when they employ resources such as frameworks (and other instruments) on the basis of their cognitive abilities to solve problems, prove theorems, shape theories, and (sometimes) to elaborate new frameworks" (33). ${ }^{5}$ The epistemology of mathematics suggested by the author employs "a certain understanding of the nature of mathematical practices oriented to the knowledge they produce" (30). It takes the mathematical community into consideration, since there is no practice without practitioners, as well as what regulates a practice, e.g. which abilities and resources these communities use. In summary, the analysis of mathematical knowledge suggested by Ferreirós is pragmatist, agent-based and historically situated.

In this paper, we start with Ferreirós' definition of mathematical practice and argue that, from that perspective, Euclidean geometry is self-sufficient and rigorous, i.e. that assumptions based in diagrams do not give rise to argument gaps as has been claimed. With that in mind, we first present, following Lassalle Casanave (forthcoming), important differences between the Euclidean practice and Hilbert's reconstructions, as well as arguments to the effect that formal reconstructions do not clarify or ameliorate (in the sense of eliminating gaps) the Euclidean demonstrative practice.

We then go on to explain why, within the Euclidean practice, diagram use is a controlled and reliable mathematical procedure. In order to do so, we turn our attention to the work of Manders (2008a, 2008b) about the systematic use of specific aspects of dia-

3 About the changes in the notion of mathematical rigor throughout the history, see Kleiner (1991). In that paper, the author claims that the patterns of mathematical rigor can vary and that it is not always the case that the changes consist in an increase in rigor. An illustrative case of rigor decrease is the use of infinitesimal calculus by Euler.

4 One example is Gauss' presentation of four distinct proofs for the Fundamental Algebra Theorem and the six distinct proofs for quadratic and higher reciprocity laws in number theory (examples taken from Ferreirós 2016, 10). A more detailed study about these issues can be found in Lemmermeyer (2000) and Goldstein et al. (2007).

5 On the appearance and motivations of the philosophy of mathematical practice, as well as a good source of important references within that tradition, see Mancosu (2008) and Giardino (2017). 
grams as a basis of inferences in that practice. We complement Manders' analysis with a tentative list of abilities and competences involved in the skilled use of diagrams within the Euclidean tradition. The result of our discussion is that the Euclidean practice is much more regimented and constrained than its critics have supposed.

The previous discussion might make it seem as if the abilities required for diagram-based reasoning in the Elements can only be acquired by a long process of specialization. While we do not deny that training and learning are crucial steps in the formation of a good geometer, we finish the paper by reviewing recent experimental results suggesting that the cognitive basis underlying diagram use in the Euclidean practice is much more basic than what one would expect. Indeed, these results show that crucial competences required for diagrammatic reasoning in the Euclidean practice, such as the recognition of topological relations between figures, are present in populations that do not even have words for most spatial or geometrical concepts. This allows us to claim that, not only the Euclidean diagrammatic practice is reliable, it is also rooted in cognitive abilities that are possessed independently of factors such as formal education or training in geometry. We conclude by raising some questions for further investigation.

\section{The Elements and formal reconstructions}

Ferreirós (2016, chap. 5) claims, against commentators (especially mathematicians and philosophers from the late nineteenth and early twentieth centuries), that the subject matter of Euclidean geometry is distinct from that of Hilbert's reconstructions. According to Ferreirós, the appearance of complete continuity between those two frameworks obscures the fact that they showcase distinct ways of doing mathematics. It is an anachronism to assess the merits of Euclidean geometry through the particular objectives and practices of modern axiomatic theories.

In this section, we intend to argue that interpreting the formal system presented in Hilbert's (1980) Foundations of Geometry (henceforth, Foundations) as if it were an amelioration (in the sense of filling gaps) of the geometry presented in the Elements is a mistake. In order to do this, we will list and discuss some important differences between these two ways of doing mathematics. We will show that the Euclidean geometry should be assessed via its own symbolic and theoretical frameworks, as well as by means of the specific competences that a geometer is expected to have if she is to be capable of rigorously working with diagram-based proofs inside of that practice.

When we look at the first six books of the Elements, for example, one finds a theory of plane geometry and instructions for its demonstrative practice (via the postulates of Book I, aitêmata, the common notions, koinai ennoiai, the symbolic framework with diagram-based reasoning, the letters linking text to diagram, the concepts expressed by the definitions etc.). The nature of the axioms and postulates within those two practices is radically different, as well as the methods of proof construction.

Building on Lassalle Casanave's (forthcoming) recent work, these are some differences between Euclid and Hilbert's frameworks that we take to be important:

1. In Euclid, the theoretical language (e.g. of the definitions) is sharply distinguished from the practical one (e.g. of the postulates), as well as theorems from problems. 
In Hilbert, practical propositions are reduced to theoretical ones by means of axioms and theorems.

2. In Euclid, the theory provides its objects by means of postulates or constructions. The construction postulates and, generally speaking, the problems themselves help achieve that end. Ontological questions fall outside the scope of this paper, but it is important to emphasize that the objects of Euclid's study are intra-theoretical - they are either constructed by means of postulates or problems and have their properties demonstrated by the theorems. We also observe that some of these objects' properties are extracted directly from the diagram, e.g. that two straight lines intersect in one (and only one) point. In the next section we will claim that it is a part of the geometer's ability within that practice to be able to recognize such aspects. ${ }^{6}$ Hilbert, on the other hand, considers three sets of elements (called points, lines and planes), but, although his axioms do assert the existence of these objects, Hilbert is only concerned with the relations between them, whatever their nature turn out to be. In other words, the objects modeled by Hilbert's system are not given nor constructed in the same sense as Euclid's; on the contrary, the former are extra-theoretical in the sense that Hilbert's system is not focused on investigating and demonstrating their specificities, but only the relations between them. This point is specially clear in a letter addressed to Frege where Hilbert explains what he thinks a formal theory is: "But it is surely obvious that every theory is only a scaffolding or schema of concepts together with their necessary relations to one another, and that the basic elements can be thought of in any way one likes" (Frege $1980,42)$. $^{7}$

3. The Euclidean distinction between solving a problem by means of a construction and demonstrating the soundness of that solution disappears in Hilbert's reconstruction, where the only activity is the demonstration of existential theorems.

4. Hilbert's axioms replace diagrams as sources of justification. The figures used by Hilbert are only pedagogical tools. All proofs must be based on pre-established axioms. ${ }^{8}$

6 As was observed by an anonymous referee, some properties of Euclidean objects are tacitly given and never made explicit by the text. However, we believe it is a part of the abilities and competences of a geometer being able to recognize the diagrammatically relevant aspects of a drawing and being able to use them in the argument. This will become clearer when we introduce Manders' distinction between the diagrammatic and the textual aspects of an Euclidean proof. In any case, see Giaquinto (2011) for a discussion of the thesis that some properties of the Euclidean space are simply assumed.

7 This conception of formal theories was given by Hilbert in the context of the Foundations but it also applies to his posterior works where theories are formal in the purely syntactic sense. The open interpretation of the primitive symbols (point, line, plane) paves the way for the appearance of strange objects, e.g. ones that do not obey the Euclidean relations. For example, if the axiom corresponding to the side-angle-side congruence criterion is substituted for a weaker one, we get a geometry where isosceles triangles do not need to have equal base angles (Euclid's proposition I.4) (Appendix II of the Foundations). This topic deserves more investigation (see, for example, Mueller 1981, and Giovannini 2013).

8 On the second section of chapter 4, Lassalle Casanave (forthcoming) shows why, more than the others, Hilbert's axiom of continuity is connected to the idea that diagrams are dispensable. 
In his later writings, circa 1920, Hilbert presents an axiomatic theory that comprises a linguistic system, i.e. a symbolic framework composed of formulas and inscriptions (inference rules) devised for passing from formula to formula inside that language. His goal was to analyze the deductive structure and independence of mathematical theories and to prove their consistency: a metamathematical concern. His method consisted in reducing mathematical theories to formal systems and then treating its proofs as mathematical objects in themselves. By doing that, one would be able to see e.g. whether any of those proofs contains a contradiction. Having that very specific objective in mind, Hilbert defined mathematical proofs in terms of formal syntactic proofs. ${ }^{9}$ However, Hilbert did not intend that definition to be taken as a general account of mathematical proofs; it was conceived as part of a more general strategy related to his foundational program. The unrestricted identification between proof and formal syntactical proof was in large measure a posterior product made by philosophers of mathematics and some logicians and mathematicians during the twentieth century. ${ }^{10}$ This identification is largely responsible for the idea that Euclidean proofs are, strictly speaking, not proofs.

Regardless of that, it is often not even clear in which sense the word 'formal' is being employed in these contexts. Lassalle Casanave (forthcoming) identifies three senses in which that word has been used in association with proofs. The sense being used by Hilbert is that of diagram-independence. A second one is that of exclusively syntactic (a special case of being free of diagrams, many times seen as the only one). A third sense of 'formal' is that of dealing with relations between objects where these objects can be conceived as any object satisfying the theory's axioms (in opposition to Euclid's theory where its objects required construction and had their properties demonstrated). Formalization in the second sense is defined inside Hilbert's Program but does not even apply to the proofs showcased in the Foundations, since the latter's proofs are not purely syntactic. ${ }^{11}$ A completely logical (thus syntactical) reconstruction of Euclidean geometry was only presented by Tarski, who employs a purely logical vocabulary and two primitive symbols to represent the extra-logical geometrical relations of in-betweenness and equidistance. In other words, there is a difference between the formal geometrical theory presented by Hilbert in the Foundations, which is formal in the senses one and three, and Tarski's theory, which is formal in the three senses. ${ }^{12}$ Lassalle Casanave concludes that we should distinguish between the notion of a proof and of a formal proof in the syntactic sense: proofs are epistemological and cog-

9 For a discussion of the functions of symbols in Hilbert's formalism, see Lassalle Casanave (2015).

${ }^{10}$ In the area of logic, see, for example, the logico-syntactical formalization of Euclidean geometry presented by Tarski \& Givant (1999). In the area of mathematics, one example of formalism that gives a great deal of attention to the presentation of the formal proofs can be found in the works of Landau in analysis (see the collected works of Edmund Landau (Heath-Brown 1989)). We would like to thank an anonymous referee for illuminating us on this point by means of very useful suggestions.

11 About Hilbert's works in the 1920's and Hilbert's Program, see Sieg (2014).

12 Tarski had paid great attention to logico-syntactical formalizations of mathematics. Even though this is outside the scope of the present paper, we would like to call attention to the role of increasing logical sophistication inside this story, a topic which deserves further historical investigation. About this point, besides Tarski's own works, we suggest Pieri's work on the foundations of geometry (see Pieri's collected works, 1980, under the title Opere sui fondamenti della matematica). 
nitive objects "whose clarification cannot be reduced to the notion of a formal proof, that is, to an aspect of a purely logical order" (forthcoming, 119).

Considerations like these point to the fact that Euclid's Elements and the modern mathematical axiomatizations are based on distinct symbolic and theoretical frameworks. ${ }^{13}$ A difference in symbolic framework means a difference in the manner of representing problems, solutions and proofs within a mathematical practice (by means of pictographic signs, diagrams, functions etc.). It is, in other words, a difference in the system of representation of the theory itself. The importance of that difference should not be underestimated. If one is not merely interested in the results established by a mathematical practice but also by the forms of reasoning by means of which those results are established, one needs to put the particular system of representation employed in the center of one's investigation. Equally important, a difference in theoretical framework entails a difference in the statements accepted by agents at a given time, the allowed forms of reasoning, and the questions and conjectures that a mathematical practice departs from.

These observations count heavily against the misguided idea that Hilbert's formal reconstruction is simply an amelioration of Euclid's Elements. There are, to be sure, historical and, up to some point, theoretical continuities between them, but a difference between mathematical practices and the frameworks which constitute them must not be overlooked. Here is, we suggest, a better way to understand the relation between these mathematical practices: the axiomatization of geometry found in Hilbert is a presentation of the results of Euclidean geometry within a distinct framework. ${ }^{14}$ What is most important about that proposal is to downplay the idea that a reconstruction of an ancient mathematical theory in a modern framework leads to the clarification - or even an analysis of - such crucial mathematical notions such as that of demonstration or mathematical knowledge. A clarification of these notions can not be made in isolation of specific mathematical practices and their symbolic and theoretical frameworks. Instead, the philosopher should ask which abilities and competences a mathematical tradition, such as Euclid's, would require of a practitioner.

When we take a closer look at distinct practices of mathematical demonstration, both Euclidean plane geometry and its axiomatic counterparts come out as self-sufficient mathematical theories on their own. They are distinct theories which exploit, in different ways, the capacities of mathematical agents, each with their own methods and merits (Ferreirós 2016, 119). The hypothesis is that the Euclidean diagram-based practice itself shows that there are no gaps in its demonstrations. Instead, it presents us with a fruitful and perspicuous way of proving results by using diagrams. As Manders says:

Euclidean diagram use forces us to confront mathematical demonstrative practice, in a much richer form than is implicit in the notions of mathematical theory and formal proof on which so much recent work in philosophy of mathematics is based; and to confront rigorous demonstrative use of non-propositional representation. The philosophical opportunities are extraordinary. (2008a, 68)

\footnotetext{
13 About the notion of symbolic and theoretical frameworks, see Ferreirós (2016, chap. 3).
}

${ }^{14}$ For more on this idea, see Lassalle Casanave and Panza (2015) 
The possibility that non-propositional resources be used in legitimate and rigorous proofs affords us a richer understanding of the notion of demonstration and mathematical knowledge. From that perspective, if one is concerned with Euclidean geometry, the right question to ask is: inside Euclid's framework, how should one proceed in order to justify inferences on the basis of a diagram? In our view, the ability and competence in dealing with diagrammatical reasoning is a key factor for answering that question. With this in mind, in the next section we examine what kind of diagrammatic elements are considered in Euclidean demonstrations and point out some abilities involved in the diagrammatic reasoning present in this practice.

\section{Diagrammatic reasoning in the Elements}

As one can see already in the first proof in the Elements (I.1), a great part (if not all) of Euclidean demonstrations depend not only on what is textually said (in the definitions, common notions and postulates) but also on steps extracted from the drawings themselves. According to Barwise \& Etchemendy (1996), even though "the possibility of error [in diagrammatic reasoning] is real, [...] it is not more serious than the types of fallacies that can occur in purely linguistic forms of reasoning" (8). In relation to the latter, the risk of fallacy has been taken care of through investigation of the problematic cases and a development of a more sophisticated understanding of linguistic proofs. The same has not been done for the former, although "it is not obvious that an analogous study of diagrammatic reasoning could not lead us to an analogous understanding of the legitimate and illegitimate uses of these techniques" (Barwise \& Etchmendy, ibid).

Manders (2008a, 2008b) goes in that direction in his enlightening study of the use of diagrams in Euclidean practice. As he emphasizes, his concern is not assessing whether Euclidean geometry is a good theory, since that is something he takes for granted given its unquestionable and long-lasting success. His concern is explaining how diagrams can be legitimate tools in Euclidean proofs, contradicting the prevailing view by claiming that such use is fundamentally controlled and safe.

Central to Mander's analysis is his influential distinction between exact and co-exact aspects of the proof. The first is the information which is dependent on the textual part of the proof (justified by definitions, postulates, common notions). They are metric information. They include, for example, equality of segments, angles or other magnitudes, congruence relations and proportionalities. The latter are aspects of the diagram which are stable under small variations of the diagram and not eliminable by perfecting it, such as part-whole relations of regions and intersection points. An example of a co-exact aspect appears already in demonstration I.1: as we draw circles on the constructive part of the proof and 'see' that they intersect at a point (Fig. 1). The existence of that intersection point is a co-exact aspect that we learn from inspecting the drawing itself. In contrast to the co-exact aspects, exact aspects cannot be reliably extracted from the diagrams, and, even though the diagrams might suggest them, they do not survive under small variations of the drawing.

A key observation by Manders is that Euclid's diagrams are used only as a source of co-exact information. Euclid never infers exact information from the drawing: 
Typical alleged 'fallacies of diagram use' rest on taking it for granted that an-to the eye apparently realized but false-exact condition would be read off from a diagram; but the practice never allows an exact condition to be read off from the diagram. Typical 'gaps in Euclid' involve reading off some explicit co-exact feature from a diagram; and this is permissible (..). (2008b, 91)

\section{Proposition 1}

On a given finite straight line to construct an equilateral triangle.

Let $\mathrm{AB}$ be the given finite straight line.

Thus it is required to construct an equilateral triangle on the straight line $\mathrm{A} \mathrm{B}$. With centre $A$ and distance $A B$ let the circle $\mathrm{BCD}$ be described; [Post. 3] again, with centre $B$ and distance $B A$ let the circle ACE be described; [Post. 3] and from the point $C$, in which the circles cut one another, to the points $\mathrm{A}, \mathrm{B}$ let the straight

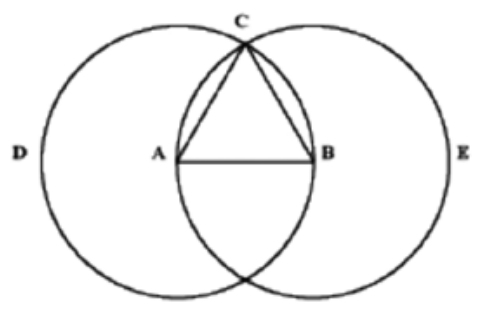
lines CA, CB be joined. [Post. I]

Now, since the point $A$ is the centre of the circle $C D B, A C$ is equal to AB. [Def. 15]

Again, since the point $B$ is the centre of the circle CAE, BC is equal to BA. [Def. 15] $\mathrm{AB}$

But $C A$ was also proved equal to $A B$; therefore each of the straight lines $C A, C B$ is equal to

And things which are equal to the same thing are also equal to one another, [C. N. I] therefore $\mathrm{CA}$ is also equal to $\mathrm{CB}$.

Therefore the three straight lines $\mathrm{CA}, \mathrm{AB}, \mathrm{BC}$ are equal to one another. ght line $\mathrm{AB}$.

Therefore the triangle $\mathrm{ABC}$ is equilateral; and it has been constructed on the given finite strai-

(Being) what it was required to do.

\section{Figure 1}

Euclid, Proposition I.1 (Heath 1968, 241-242).

Thus, diagram use is rigorous and controlled because diagrams are a reliable source of co-exact information. In cases where it is not clear whether some aspect of a diagram is co-exact, we can settle the question by deforming or perfecting the diagram and checking whether the relevant information displays stability. This idea is present in what Manders calls the diagram control theory (2008b), that is, the obligation a geometer has of trying out different diagrammatical configurations of the textual part of the proof, whenever there is some doubt. ${ }^{15}$ Manders' analysis renders clear under which conditions it is legitimate in Eu-

15 Among the examples offered by critics as evidence that diagram use is not reliable, we find the famous fallacy such that "every triangle is isosceles". This example is discussed in recent analysis of authors such as Norman (2003) and Manders (2008b), where the authors try to show why it does not amount to a criticism of Euclidean proofs. The point is that every time that the fallacious proof is exhibited, 
clidean practice to use diagrammatic information in an inference. In other words, Manders makes explicit one of the competences that a Euclidean geometer must have for using diagrams efficaciously.

It is thus interesting to notice that Manders' analysis, specially his distinction between exact and co-exact aspects of Euclidean proofs, enabled the development of formal systematizations of the Elements based on the idea that Euclid's use of diagrams in geometric proofs "is not soft and fuzzy, but controlled and systematic, and governed by a discernible logic” (Avigad et al. 2009, 3). Two noteworthy examples are Miller's (2007) and Avigad et al.'s (2009) formal systematizations of the geometrical theory of the Elements. ${ }^{16}$ These two axiomatic systems strive to formalize Euclidean diagram use by transforming Manders' insights into parts of the syntax or the semantics of their logic. Interestingly enough, Miller's (2007) formal system uses diagrammatic elements as an essential part of its syntax, although his diagrams are not the same as the ones found in Euclid, but graph-theoretic objects that can undergo certain combinatorial operations with the objective of reflecting the topological (and thus, co-exact) aspects of Euclid's figures. In Avigad et al, on the other hand, the co-exact aspects that allowed us to gain information from diagrams are transformed into a series of inference principles. Thus, whereas we had to look at the diagram to see that the two circumferences intersected at a point in proof I.1 of the Elements, in Avigad et al.'s system we are able to derive the same conclusion by means of using Rule 5 of Diagram Intersections (p. 28), which says: if point a is on circumference $\alpha$, point $b$ is in circumference $\alpha$, point $\mathrm{a}$ is in circumference $\beta$, and point $b$ is on circumference $\beta$, then $\alpha$ and $\beta$ intersect.

Notwithstanding these differences, both systems have the same objective: modeling the proof methods found in the Elements by means of a sound and complete formal system. These systems are, therefore, formal in a distinct sense than the systems presented by Hilbert or Tarski are, i.e. the former, but not the latter, preserve central characteristics of Euclidean geometry and are mostly focused on formally grounding the topological information that Euclidean diagrams present. It is thus important to realize that formalization projects are often very distinct from each other and that each can have very distinct methodologies and objectives. It is also important to emphasize that these formalizations, notwithstanding their fundamental differences, can be seen as logical analysis of the geometry presented by Euclid, but not as a clarification of the concept of demonstration employed by him. In other words, understanding how to do Euclidean geometry with diagrams will not be achieved by means of formalizing that theory. As we have been insisting, one of the great virtues of Manders' investigation is explicating the abilities involved in the legitimate use of diagrams in Euclid, and, we believe that, for the reasons presented in section 2, this is the

only one of the possible diagram configurations is provided. Norman (2003) shows that, in one of the possible configurations of the diagram, one information extracted from the figure in the fallacious proof does not appear, i.e. it is not co-exact information in Manders' terminology. Heath (1921) calls attention to a Euclid's work entitled Pseudry or Pseudographemata which we know only from secondary sources such as Proclus (who called it the Book of Fallacies). That book might have been intended to make the geometer more attuned to the possibility of avoiding fallacies such as this (Heath 1921, 430-431).

${ }^{16}$ We would like to thank an anonymous referee for calling attention to these relevant points and references. 
only way for an adequate comprehension of the concept of demonstration to be found in the Elements. We will thus follow in that direction, trying below to deepen Manders' analysis by means of investigating other competences present in the diagrams use of Euclidean geometry.

Besides the ability of recognizing and employing adequately the co-exact aspects of diagrams, a geometer must also be able to construct, manipulate and interpret a diagram adequately, and that involves seeing the diagrams' function as a species of proof artifact. Understanding diagrams as artifacts involves, we suggest, a perspective like that of Giardino's (2013), who advances "an account of reasoning in and from diagrams based on the conception of diagrams as tools used within a specific practice" (141). One important idea is that the competent use of diagrams does not require being guided by an explicit set of rules. On the contrary, mere familiarity with a practice is enough to guarantee that one manipulates diagrams with competence. According to her:

(...) in diagrammatic reasoning what counts is not the appearance of a diagram and a list of explicit rules that can be applied to it, but rather a set of procedures: when one learns to use a certain diagrammatic system for performing some inferences, she learns a manipulation practice. The diagram becomes the mathematician's worksite, where operations, plans, and experiments are made in order to find solutions and reasons for these solutions. (2013, 145-146, emphasis in the original)

Thus, an important step towards understanding the diagrammatic reasoning in Euclid is conceiving diagrams not as mere instantiations or illustrations of the proof s textual part, but as tools and instruments promoting inferences via its possibilities of manipulation. In the case of Euclidean geometry, being aware of such possibilities is, for example, knowing how to adequately apply the construction postulates (1-3). The first postulates allows for three distinct moves in a diagram: drawing a straight line in between two given points (postulate 1), extending a straight line indefinitely (postulate 2 ) and, from a point and a radius, drawing a circle (postulate 3 ). These construction rules constrain the geometer's operations in the diagrams and force the interlocutor to accept a certain set of diagrammatical movements. Learning how to construct an adequate diagram is knowing how to manipulate the diagram in accordance with textual prescriptions and learning how to recognize the co-exact aspects emerging from those manipulations.

A point to be emphasized is that Euclidean diagrams are not merely visualized, but also interpreted and manipulated according to a whole practice. Interpreting a diagram is not only about perceiving its static aspects, but considering the actions that can be performed on it and being able to identify the results that emerge. The geometer constructs and reasons with the diagram not by considering how it is visually given, but how it is conceived. For example, a line on a diagram can be conceived either as the side of a triangle or as the radius of a circle. Neither does it need to be a straight line, as long as it is sufficiently straight to be seen as a straight line. The possibility of this gestalt-shift is what explains how figures often 'pop-up' in an Euclidean proof - such as when an equilateral triangle 'pops up' in the proof I.1 of the Elements (Fig. 1) - and thus, how something new can emerge in the drawing. This ability of seeing as is employed in many Euclidean proofs -see Macbeth (2014)-.

We can see these characteristics in many of the proofs presented in the Elements. For illustration purposes, we present proof I.37 (Fig. 2). The first step of the proof consists 
in performing an action on the initially presented diagram. That is allowed by the second Postulate of construction: "prolong AD in both directions to $\mathrm{E}$ and F". The next two movements performed on the diagram are also allowed by the postulates (in that case, by the first one) and previously proved propositions (the proposition I.31): "draw BE through B parallel to CA, and draw CF through C parallel to BD". The geometer must be able to recognize the adequate actions to be performed on the diagram and whether these actions respect the restrictions by the postulates. In the next step, the geometer must be able to correctly interpret the outcome of these actions, i.e. to recognize the emergence of two figures EBCA and DBCF in the diagram and see, e.g. the straight CA (previously a side of the triangle $\mathrm{ABC}$ ) as a side of the parallelogram EBCA (the same with the straight $\mathrm{DB}$, previously interpreted as a side of the triangle $\mathrm{DBC}$ and now seen as a side of a parallelogram DBCF). These gestalt-shifts play a role in many proofs in the Elements and are an essential aspect of diagram use in mathematics. Being able to detect them and to see bits of a figure either as this or that is one of the abilities that a geometer must master.

\section{Proposition 37}

\section{Triangles which are on the same base and in the same parallels are equal to one another.}

Let $\mathrm{ABC}, \mathrm{DBC}$ be triangles on the same base $\mathrm{BC}$ and in the same parallels $\mathrm{AD}, \mathrm{BC}$; I say that the triangle $\mathrm{ABC}$ is equal to the triangle $\mathrm{DBC}$.

Let $\mathrm{AD}$ be produced in both directions to $\mathrm{E}, \mathrm{F}$; through $\mathrm{B}$ let $\mathrm{BE}$ be drawn parallel to $\mathrm{CA},[1.31$, and through $\mathrm{C}$ let $\mathrm{CF}$ be drawn parallel to $\mathrm{BD},[1.31]$.

Then each of the figures EBCA, DBCF is a parallellogram; and they are equal, for they are on the same base $\mathrm{BC}$ and in the same parallels $\mathrm{BC}, \mathrm{EF},[1.35]$.

Moreover the triangle $\mathrm{ABC}$ is half of the parallelogram $\mathrm{EBCA}$; for the diameter $\mathrm{AB}$ bisects it, [1.34].

And the triangle $\mathrm{DBC}$ is half of the parallelogram DBCF; for the diameter DC bisects it, [1.34].

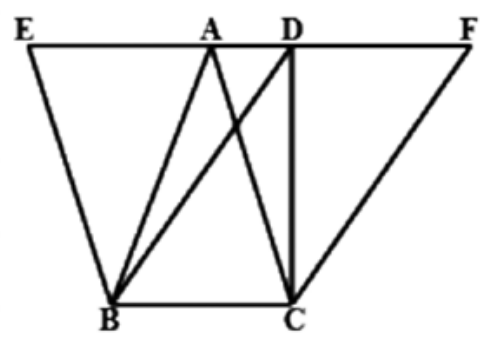
[But the halves of equal things are equal to one another.] Therefore, the triangle $\mathrm{ABC}$ is equal to the triangle $\mathrm{DBC}$.

Therefore etc.

\section{QE.D.}

\section{Figure 2}

Euclid, Proposition I.37 (Heath 1968, 332).

Thus, the geometer must be able to see the diagram in movement, imagining the possible actions that can be performed on it in order to reach a certain result and interpret it properly.

Summarizing the previous points lead us to the following tentative list of abilities required in order to use diagrams within the Euclidean practice: 
1. Knowing how to draw a diagram on the basis of textual information in a way that preserves its co-exact aspects. For example, if the text asks for a circle to be drawn, the adequate diagram must involve a symmetrical closed curve (not an open curve).

2. Knowing how to employ the co-exact aspects of a drawing in inferences and knowing how to recognize the relevant aspects of the diagram regardless of imperfections in the drawings. That is related, for example, to the willingness to try out different diagrammatical configurations of the textual part of the proof whenever there are doubts about the stability of some purported co-exact aspect.

3. Knowing the adequate method for constructing and manipulating a diagram in accordance with the three construction postulates. This ability involves being able to conceive and interpret the diagram, as opposed to merely visualizing it.

4. Being able to imagine how a diagram should be drawn, regardless of not being able to draw it perfectly.

This incomplete list takes us closer to understanding how reasoning operates within the Euclidean practice and why it is a reliable source of mathematical knowledge. As we have seen, one crucial point is that diagrams should only be used as a source of co-exact information, that is, that type of information that survives small modifications of the drawing. This explains why diagrams are reliable mathematical tools even when imperfectly drawn.

Even if our analysis shows why the use of diagrams in the Euclidean practice is rigorous, one could still pose the following criticism: "even if we accept that diagrams are a reliable and controlled source of co-exact information, what guarantees that these aspects are always recognizable?" In other words, why should it not be often the case that different people looking at the same diagram would disagree about its co-exact aspects? In order to meet this challenge, we will take recent experimental results about visuospatial reasoning into consideration and use them to investigate the cognitive basis of diagrammatic reasoning. Our main point will be that the ability to recognize co-exact properties of drawings is based on a universal trait of human visuospatial cognition. This will be the subject of the next section.

\section{Cognitive abilities in Euclidean geometry}

In this section, we argue that the use of diagrams in the Elements is rooted in cognitive abilities that are universal in the sense of being shared by populations independently of their level of formal education, training in geometry or the possession of linguistic resources to express geometrical concepts. Together with the fact-defended in the previous section-that the Euclidean use of diagrams is controlled and legitimate, these points present a strong case in favor of the Euclidean diagram-based practice's mathematical reputability.

In order to investigate the cognitive basis of diagram use in the Euclidean practice, we explore recent studies in cross-cultural geometrical cognition. Our emphasis will be 
on two particular experiments that provide empirical evidence for the existence of basic intuitions of visuospatial relations corresponding to one of the core competences involved in Euclidean geometry enumerated in the previous section: the recognition of co-exact relations in a drawing. Specifically, our interest is in experiments comparing the abilities to recognize geometric information of populations with formal education (and acquainted with tools such as compass and ruler) with populations without these characteristics. The purpose of these experiments is to verify whether some of these abilities are present in human beings independently of their culture, education and environment.

One notable experiment was performed by Dehaene et al. (2006) with an Amazonian indigenous group, the Munduruku. The reasons why this population is interesting for cognitive scientists are threefold. First, they do not possess formal education in mathematics; second, they are not familiar with the use of compass or rulers (mathematical tools essentials to Euclidean geometry); finally, they have very few words for geometrical concepts and spatial relations, as well as a limited vocabulary for numbers. Regardless of all that, cognitive scientists showed that they are still able to solve non-trivial spatial tasks. The experiment consisted in showing them screens of figures containing multiple representations of one geometrical concept or relation together with a representation of a distinct one-see figure 3 below. The experimenter then asked the participants to indicate-among the figures at the screen - the 'weird' or 'ugly' drawing. Among the geometrical concepts and relations represented, some were of topological relations (e.g. connectedness, being inside, being closed), some of metric relations (e.g. equidistance of points). Munduruku adults and children had identical accuracy in solving those tasks as Western children, while Western adults performed significantly higher. Even more relevant to our present concerns, the experiments showed that, even though the Mundurukus had performance deficits in domains which involved judgments of paradigmatically exact properties ${ }^{17}$, they "succeeded remarkably well with the core concepts of topology" (381), which include some of the most important co-exact relations exploited in Euclidean proofs, including the relation of connectedness. This could be taken as initial evidence that the ability to detect co-exact information in a diagram, one of the core abilities required by the Euclidean diagram-based practice, is present in human beings independently of education and cultural background.

Van der Ham et al. (2017) improved on the previous experiment by explicitly taking into account Manders' analysis of the Euclidean diagram-based practice and its exclusive reliance on co-exact aspects of the drawings. With that in mind, they performed a similar experiment with Senegalese and Dutch subjects with the intention of testing and comparing their abilities to recognize exact and co-exact aspects of di-

17 The Mundurukus' performance was lower but still higher-than-chance for slides dealing with paradigmatically exact properties, such as symmetries and metric properties. The two domains where the Mundurukus' performance was poor were in "a series of slides assessing geometrical transformations, for instance, by depicting two triangles in a mirror-symmetry relation; and another two slides in which the intruder shape was a randomly oriented mirror image of the other shapes" (Dehaene et al. 2006, 381). 
agrammatic representations-see figure 4 below. ${ }^{18}$ In this case, the Senegalese population was composed both by subjects with and without formal education. All the Dutch subjects, on the other hand, were educated in mathematics and were familiar with the use of compass, rulers and maps. In this experiment, they compared the results between the Dutch and Senegalese populations, and between the two different kinds of population that composed the latter. If the recognitional abilities for some types of spatial relations are indeed universal, education should not have a significant effect on performance. In accordance to that prediction, the results showed that the performances were highly similar for all participants. ${ }^{19}$ Very importantly, the difficulty profiles were almost identical for all participants, i.e. the subjects' performance varied in a similar fashion from test to test. All subjects performed significantly better in the recognition of co-exact aspects ( $~ 80 \%$ accuracy for the mixed group of Senegalese, $~ 95 \%$ for the Dutch) than in the recognition of exact aspects ( $\sim 50 \%$ for the mixed group of Senegalese, $\sim 70 \%$ for the Dutch). These results provide empirical evidence in favor of the hypothesis that the recognition and judgments of co-exact spatial relations (those prominent in Euclidean geometry) are much more reliable than the recognition and judgments of exact ones.

In summary, both experiments strongly suggest that the accuracy of co-exact information judgments is significantly higher than that of exact ones (Van der Ham et al. 2017, 274) even for populations that have not had any type of formal education or, in the case of the Mundurukus, do not even have linguistic resources to express basic geometrical concepts. These results fit particularly well with the Euclidean diagram-based practice's constraints on the type of information to be drawn from diagrams. This is precisely what allowed Euclid to minimize disagreement about his demonstrations and avoid criticisms. ${ }^{20}$ As the authors conclude, "high performances in the co-exact condition suggest an initial cognitive explanation for the reliability of judgments involving co-exact spatial relations advocated by Manders to explain why only co-exact relations are inferred from diagrams in Euclid's deductive reasoning" (Van der Ham et al. 2017, 277). This seems to suggest that the Euclidean diagram-based practice is not only epistemologically reliable but also rooted in basic cognitive abilities.

${ }^{18}$ Van der Ham et al's experiment was in many senses a realization of the suggestions put forward by Hamami \& Mumma (2013).

${ }_{19}$ Nevertheless, the Dutch participants showed higher accuracy across the tests than the other participants. The authors contend that this difference cannot be explained by the two groups' distinct average levels of formal education, since the Senegalese population without formal education had a highly similar performance to the Senegalese population that had received basic mathematical training relevant to the tasks at hand. Instead, the authors argue that the difference in performance between the two nationalities must be due to "general cultural differences in how people use spatial information in their daily activities" (Van der Ham et al. 2017, 277). For our present concerns, it is more important to emphasize that all of the participants' difficulty profiles were very similar.

${ }^{20}$ About Euclid's concerns in minimizing disagreement and improving the reliability of his proofs, see Proclus (1970). 
Closure
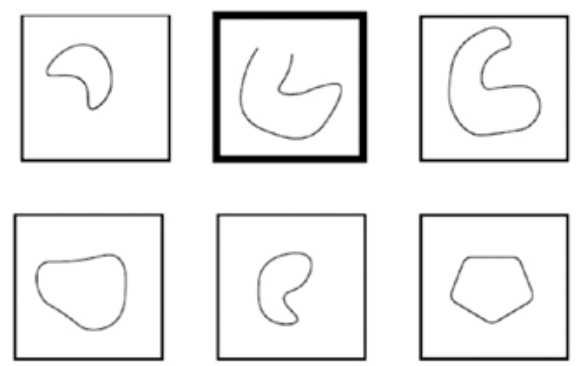

Equidistance
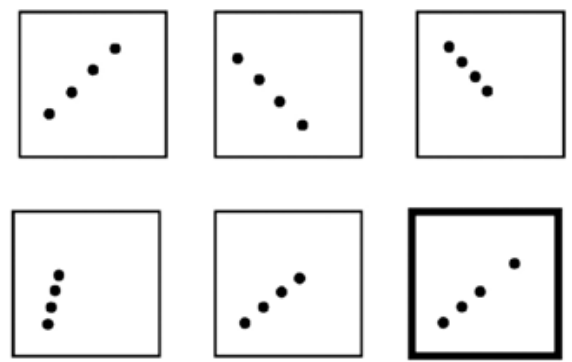

Figure 3

In this task, the subjects have to find the "weird" or "strange" figure among the six slides. Pictures adapted from (Dehaene et al. 2006, 382).

\section{Co-exact}
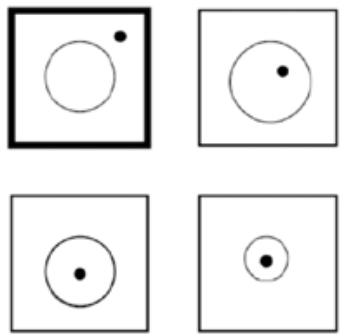
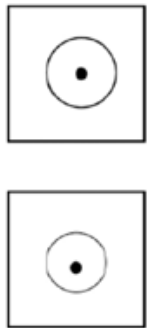

Exact
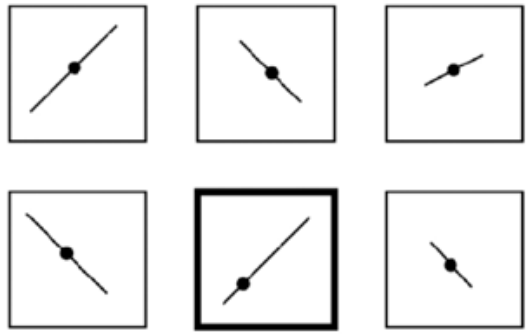

\section{Figure 4}

As in the above-mentioned study, in these tasks the subjects have to find the "weird" or "strange" picture among the six slides. Pictures adapted from (Van der Ham et al. 2017, 272).

It is important to pause at this point and emphasize the following point: one should not infer from the results of these experiments that abstract geometrical knowledge, such as that presented in the Elements, emerges in a completely natural way from humans' cognitive architecture. There is a significant gap between the kinds of basic cognitive competences involved in the recognition of exact and co-exact features of a drawing (those dealt with by the experiments) and the kind of knowledge born out of a sophisticated mathematical practice such as Euclidean geometry. Dehaene et al. (2006) seem to make little of this point when they claim, for example, that "our experiments [...] provide evidence that geometrical knowledge arises in humans independently of instruction, experience with maps or measurement devices, or mastery of a sophisticated geometrical language" $(384){ }^{21}$

21 According to authors like Spelke et al. (2010), these experiments demonstrates the existence of a "natural geometry", i.e. a kind of geometric cognition that emerges spontaneously in human beings, independently of the cultural context from which they come. 
We want to insist that the notion of geometrical knowledge should not be used so lightly. In order to be a competent agent in Euclidean geometry, one must possess abilities that go far beyond the abilities which the aforementioned experiments were concerned with. As we have sketched in the previous section, a skilled geometer must possess many competences which are only learned by means of the Euclidean practice itself and which would not emerge irrespective of that. Euclidean geometry is a theoretical framework with idealizations and hypotheses that go way beyond our spontaneous perception of space and figures. The Elements has an internal structure that emphasizes the very abstract character of the problems that it tries to solve. It employs sophisticated demonstrations, some of them very distant from day-to-day geometrical reasoning, such as the method of reductio ad absurdum. Besides all of that, Euclid's work presupposes very significant idealizations (e.g. points without extension) that are necessary for the establishment of concepts and geometric figures which, given their idealized nature, will inevitably resemble only weakly the objects that we know by means of ordinary spatial cognition (Gaber \& Schlimm 2015, 362-363; Ferreirós \& García-Perez 2018). We emphasize that geometry is not a static body of knowledge developed spontaneously, but a joint product of evolution and the cultural achievements of a population, such as material tools and symbolic representations. ${ }^{22}$ Thus, although we agree that the aforementioned experiments establish the reliability of judgments based on co-exact information - that is, they show how those judgments are grounded in very basic cognitive abilities one should be careful not to go from there to the stronger claim that geometrical knowledge itself is universal and culturally independent. ${ }^{23}$

We conclude this section with a suggestion for future investigation. It seems that, if one wants to understand our geometrical capacities, then it would be illuminating to carve out distinct levels of complexity. In section two of this paper, we analyzed the abilities learned inside of a specific geometric practice. More particularly, in that section, we focused on the abilities particular to the Euclidean framework such as the capacity of cor-

22 Overmann (2013), for example, is a cognitive archaeologist who saw, with ethnographic supportquoted the sources in her paper-, that populations with low level of material complexity (size population, social classes, religion, and so on) did not (and typically do not) develop a number system to count beyond five. We do not know of any study on the relations between material complexity and geometry, but we think it is fair to believe that the results would be comparable to these of Overmann's.

${ }^{23}$ A view that is analogous to ours has been defended, with the support of empirical results, for the related case of numerical cognition (Núñez 2011; 2017). In these works, the author distinguishes between (what he calls) quantical cognition from (properly) numerical cognition. Both are cognitive abilities having to do with the recognition of quantities, however, the former differs from the latter in that it is a mere "inexact and non-symbolic" (Núñez 2017, 404) capacity for discriminating quantities, being detectable even in primates and pre-linguistic infants, while the latter is essentially symbolic, allowing for complex processes such as general exact quantification. Núñez's thesis is that the former type of cognition is a biologically evolved capacity that is innate at least in the sense that it is not learned and not contingent on a particular culture. The latter, on the other hand, "demands crucial ingredients-cultural traits: specific cultural concerns and practices, and the use of symbolic reference" (ibid, 405). In summary, having the capacity for quantical cognition is necessary but not sufficient for having the capacity to engage in full-blown numerical cognition such as arithmetical reasoning. 
rectly applying the postulates of construction, manipulating the diagrams and correctly interpreting its outcomes. In the present section, we focused on abilities pertaining to a more basic level, that of recognizing visuospatial aspects from geometric configurations, and claimed that this ability grounds the reliable recognition and use of co-exact aspects of diagrams.

We believe that a richer analysis of the cognitive development of abstract geometrical knowledge needs to consider an intermediary step in between the biologically evolved capacities of the basic level and the abilities proper to specific geometrical frameworks. In other words, it needs to demarcate a level where cultural ingredients - such as the introduction of symbolic frameworks by means of, for example, the use of maps - come together with our more basic capacities and pave the way for the posterior arrival of abstract geometrical knowledge. ${ }^{24}$ In other words, it should consider the intersection between nature and culture: not only the pre-existent cognitive capacities but also the remarkable influence of specific cultural practices and their use of cognitive tools. In our view, progress in that subject will take us closer to understanding how reasoning operates within the Euclidean practice. This could be the basis for a philosophical view opposing the traditional one according to which diagrammatical proofs are unreliable. ${ }^{25}$

\section{Final considerations}

In this paper we have opposed the traditional view of diagram use in proofs and have argued that they can be mathematically rigorous tools of geometric reasoning. We focused mainly in the particular case of Euclidean geometry, where diagrams are commonly used as source of information in proof steps. Our case in favor of the Euclidean practice is twofold. First, we showed that the use of diagrams within that practice is strictly regimented so as not to give rise to errors. Second, we complemented our analysis of diagram use by reviewing recent experimental results purporting to show that some important abilities over which the Euclidean diagram-based practice is based are universal traits of human spatial cognition.

We started by showing that the traditional view culminates in a conception of proof as formal proof, one in which diagrams cannot play any significant role. But, as we intend to have argued, this view does not stand by itself when we conceive of mathematical knowledge as a product of different historically situated mathematical practices. We argued that, from that perspective, Euclidean geometry is self-sufficient and rigorous, that the use of di-

${ }^{24}$ The recent literature already shows signs of heading in that direction, especially in cognitive archaeology studies on the development of mathematics (de Cruz, 2012; Overmann, 2013; 2016). Similarly, Keller (2004) distinguishes three periods from geometry's arcaheo-ancestral origins until the appearance of Euclidean geometry. In that sense, it is important to take into account some recent studies in the area of Cultural Evolution (Laland 2017), and, more particularly, in evolutionary and cognitive archaeology (Bruner y Lozano 2014 a, 2014 b; Laland \& O’Brien 2010, and Coolidge and Wynn, 2016) that could be useful to understand the archaeo-cognitive genesis of proto-mathematics, and its relation with later mathematical developments.

25 See Giaquinto (2007). 
agrams is controlled and, consequently, legitimate as a source of inferences. With that in mind, we structured this paper in three sections which we can summarize as follows:

1. In the first section, we contrasted Hilbert's geometry (exhibited in the Foundations) with Euclid's and argued that it is an error to evaluate the latter through the merits of the former. Formal reconstructions cannot be lauded as ameliorationsin the sense of correcting gaps - of the Euclidean practice, since they have distinct symbolic and theoretical frameworks. We concluded the section by suggesting that a philosophical analysis of mathematical knowledge needs to be sensitive to the specific mathematical practices with which one is concerned. In this sense, the philosopher should ask oneself which abilities and competences a mathematical tradition, such as Euclid's, would require of a practitioner.

2. In the second section, we explored the abilities and competences required for engaging in the diagram-based reasoning presented in the Elements-one of the key factors for understanding why the Euclidean practice has been so successful and reliable. With that in mind, we followed Manders in arguing that the Euclidean diagrammatic practice only licenses the extraction of co-exact information from diagrams. We took this to show why the Euclidean diagram use is rigorous. In the remainder of the section we presented and explored other abilities that we believe to be equally important for a Euclidean geometer, such as the abilities of constructing, manipulating and interpreting a diagram adequately. Under this perspective, the diagram should be seen as an artifact on which the geometer makes operations, experiments and performs inferences on the basis of the restrictions of the Euclidean framework.

3. In the third section, we complemented our analysis of Euclidean diagram use by taking into account recent experimental results according to which the judgments of co-exact relations is much more reliable than that of exact ones, independently of one's level of formal education or training in geometry. This fits very well with the fact that Euclid exclusively relied on these aspects in his diagram-based proofs. As we have emphasized, this does not allow us to make the stronger claim that Euclidean geometry emerges spontaneously from human beings' cognitive architecture. Instead, our hypothesis is that classical geometry is grounded on basic perceptual abilities which are then carefully developed and regimented inside of a shared framework. We closed the paper with some suggestions for future investigation.

\section{REFERENCES}

Avigad, Jeremy, Edward Dean and John Mumma. 2009. A formal system for Euclid's elements. Review of Symbolic Logic 2/4: 700-768.

Barwise, Jon and John Etchemendy. 1996. Visual information and valid reasoning. In G. Allwein \& J. Barwise, eds., Logical reasoning with diagrams, 179-200. Oxford: Oxford University Press.

Borwein, Jonathan. 2008. Implications of experimental mathematics for the philosophy of mathematics. In B. Gold \& R. A. Simons, eds., Proof and other dilemmas: Mathematics and Philosophy, 33-60. Washington: Spectrum/ The Mathematical Association of America.

Bruner, Emiliano and Marina Lozano. 2014a. Extended mind and visuo-spatial integration: three hands for the Neandertal lineage. Journal of Anthropological Sciences 92: 273-280. 
Bruner, Emiliano and Marina Lozano. 2014b. Three hands for the Neandertal lineage: reply to the comments. Journal of Anthropological Sciences 92: 303-305.

Carnap, Rudolf. 1939. Foundations of logic and mathematics. In International Encyclopedia of Unified Science I/3. Chicago: University of Chicago Press.

Church, Alonzo. 1956. An introduction to mathematical logic. Princeton: Princeton University Press.

Coolidge, Frederick L. and Thomas Wynn. 2016. An introduction to cognitive archaeology. Current Directions in Psychological Science 25/6: 386-392.

de Cruz, Helen. 2012. Are numbers special? Cognitive technologies, material culture and deliberate practice. Current Anthropology 53: 215-216.

Dedekind, Richard. 1963. Continuity and the irrational numbers. In W. Beman, trans., Essays on the theory of numbers, 1-27. New York: Dover Publications.

Dehaene, Stanislas, Véronique Izard, Pierre Pica and Elizabeth Spelke. 2006. Core knowledge of geometry in an Amazonian indigene group. Science 311/5759: 381-384.

Ferreirós, José. 2016. Mathematical knowledge and the interplay of practices. Princeton: Princeton University Press.

Ferreirós, José and Manuel J. García-Pérez. 2018. ¿"Natural” y "Euclidiana”? Reflexiones sobre la geometría práctica y sus raíces cognitivas", Theoria 33/2: 325-344.

Frege, Gottlob. 1980. Philosophical and mathematical correspondence. In G. Gabriel, H. Hermes, F. Kambartel, C. Thiel, \& A. Veraart, eds., Gottlob Frege: Philosophical and mathematical correspondence, 31-52. Oxford: Basil Blackwell.

Gaber, David and Dirk Schlimm. 2015. Basic mathematical cognition. Wiley Interdiscip. Rev. Cogn. Sci. 6/4: 355-369.

Giardino, Valeria. 2013. A practice-based approach to diagrams. In M. Aminrouche \& S. Shin, eds., Visual reasoning with diagrams, 135-151. Basel: Birkhäuser.

Giardino, Valeria. 2017. The practical turn in philosophy of mathematics: a portrait of a young discipline. Phenomenology and Mind 12: 18-28.

Giaquinto, Marcus. 2007. Visual thinking in mathematics. New York: Oxford University Press.

Giaquinto, Marcus. 2011. Crossing curves: a limit to the use of diagrams in proofs. Philosophia Mathematica 19/3: 281-307.

Giovannini, Eduardo. 2013. Hilbert y la fecundidad matemática del método axiomático. Notae Philosophicae Scientiae Formalis 2/4: 149-163.

Goldstein, Catherin, Norbert Schappacher and Joachim Schwermer, eds. 2007. The shaping of arithmetic after C. F. Gauss's Disquisitiones Arithmeticae. Berlin, Heidelberg, New York: Springer.

Hallett, Michael and Ulrich Majer. 2004. David Hilbert's lectures on the foundations of geometry, 1891-1902. Berlin, Heidelberg, New York: Springer.

Hamami, Yacin and John Mumma. 2013. Prolegomena to a cognitive investigation of Euclidean diagrammatic reasoning. Journal of Logic, Language and Information 22/4: 421-448.

Heath, Thomas L. 1921. A history of Greek mathematics, Vol. 1: From Thales to Euclid. New York: Dover.

Heath, Thomas L. 1968. The thirteen books of the elements, Vol. I. Books I and II. Cambridge: Cambridge University Press.

Heath-Brown, D. R. 1989. Edmund Laudan: collected works. Bulletin of the London Mathematical Society 21/4: 342-350.

Hilbert, David. 1980. Foundations of geometry. La Salle, Illinois: Open Court.

Keller, Olivier. 2004. Aux origines de la géométrie: Le paléolithique et le monde des chasseurs cueilleurs. Paris: Vuibert.

Kleiner, Israel. 1991. Rigor and proof in mathematics: A historical perspective. Mathematics Magazine 64/5: 291-314.

Laland, Kevin and Michael J. O’Brien. 2010. Niche construction theory and archaeology. Journal of Archaeological Method and Theory 17/4: 303-322.

Laland, Kevin. 2017. Darwin's unfinished symphony. How culture made the human mind. Princeton \& Oxford: Princeton University Press. 
Lassalle Casanave, Abel (forthcoming). Por construção de conceitos: em torno da filosofia kantiana da matemática. Rio de Janeiro: PUC-Rio.

Lassalle Casanave, Abel. 2015. On some functions of symbols in Hilbert's formalism. In E. Hermann Haeusler, W. de Campos Sanz \& B. Lopes, eds. Why is this a Proof? Festschrift for Luiz Carlos Pereira, 5-14. London: College Publications.

Lassalle Casanave, Abel and Marco Panza. 2015. Pruebas entinemáticas y pruebas canónicas en la geometría plana de Euclides. Revista Latinoamericana de Filosofía 41/2: 147-170.

Legris, Javier. 2012. Nota sobre el concepto de demostración en C.S. Peirce. Notae Philosophicae Scientiae Formalis 1/2: 124-134.

Lemmermeyer, Franz. 2000. Reciprocity laws: From Euler to Eisenstein. Berlin: Springer.

Macbeth, Danielle. 2014. Realizing reason: A narrative of truth and knowing. Oxford: Oxford University Press.

Mancosu, Paolo, ed. 2008. The philosophy of mathematical practice. New York: Oxford University Press.

Mancosu, Paolo. 2005. Visualization in logic and mathematics. In P. Mancosu, K.F. Jørgensen and S.A. Pedersen, eds. Visualization, explanation and reasoning styles in mathematics, 13-30, Dordrecht: Springer.

Manders, Kenneth. 2008a. Diagram-based geometric practice. In P. Mancosu, ed. The philosophy of mathematical practice, 65-79. New York: Oxford University Press.

Manders, Kenneth.2008b. The Euclidean diagram. In P. Mancosu, ed. The philosophy of mathematical practice, 80-133. New York: Oxford University Press.

Miller, Nathaniel. 2007. Euclid and his twentieth century rivals: Diagrams in the logic of Euclidean geometry. Stanford: CSLI Publications.

Mueller, Ian. 1981. Philosophy of mathematics and deductive structure in Euclid's Elements. Cambridge (MA): MIT Press.

Norman, Alexander Jesse. 2003. Visual reasoning in Euclid's geometry: An epistemology of Diagrams. Philosophy PhD, University College London.

Núñez, Rafael. 2011. No innate number line in the human brain. Journal of Cross-Cultural Psychology 42/4: 651-668.

Núnez, Rafael. 2017. Is there really an evolved capacity for number? Trend in Cognitive Science 21/6: 409424.

Overmann, Karenleigh. 2013. Material Scaffolds in numbers and time. Cambridge Archaeological Journal 23/1, 19-39.

Overmann, Karenleigh. 2016. Materiality and numerical cognition: A material engagement theory perspective. In T. Wynn \& F. Coolidge, eds., Cognitive models in palaeolithic archaeology, 89-112. Oxford: Oxford University Press.

Pieri, Mario. 1980. Opere sui fondamenti della matematica. Bologna: U.M.I.

Proclus. 1970. A commentary on the first book of Euclid's Elements. Translated by Glenn R. Morrow. Princeton: Princeton University Press.

Russell, Bertrand.1919. Introduction to mathematical philosophy. Lodon: Routledge.

Russell, Bertrand. 1949 [1901]. Mathematics and the metaphysicians. In B. Russell, Mysticism and logic and other essays, 74-96. London: George Allen and Unwin LTD.

Sieg, Wilfried. 2014. The ways of Hilbert's axiomatics: structural and formal. Perspectives on Science 22/1: 133-158.

Spelke, Elizabeth, Sang Ah Lee and Verónique Izard. 2010. Beyond core knowledge: Natural geometry. Cognitive Science 34/5: 863-884.

Tarski, Alfred and Steven Givant. 1999. Tarski's system of geometry. The Bulletin of Symbolic Logic 5/2: 175-214.

Tennant, Neil. 1986. The withering away of formal semantics? Mind and Language 1/4: 302-318.

Van der Ham, Ineke. J. M., Yacin Hamami and John Mumma. 2017. Universal intuitions of spatial relations in elementary geometry. Journal of Cognitive Psychology 29/3: 269-278. 
Tamires Dal Magro holds a BA and MA in Philosophy from the Universidade Federal de Santa Maria, with a specialization in the Philosophy of Science. Presently, she is a PhD candidate in Philosophy at the Universidade Estadual de Campinas (UNICAMP) with a scholarship from the Fundação de Amparo à Pesquisa do Estado de São Paulo (FAPESP). During 2017 she was a visiting researcher at the department of Philosophy and Logic of the Universidad de Sevilla. Her current research is focused on the philosophy of mathematics, with more specific interests in mathematical practices, Euclidean geometry, symbolic knowledge, diagrammatic reasoning and heterogeneous proofs.

AdDress: Instituto de Filosofia e Ciências Humanas. Universidade Estadual de Campinas (UNICAMP). R/ Cora Coralina, 100, 13083-896, Cidade Universitária Zeferino Vaz, Barão Geraldo, Campinas, São Paulo, Brasil. E-mail: tamiresdma@gmail.com

Manuel J. García-Pérez is a PhD candidate at the department of Philosophy and Logic of the Universidad de Sevilla, under the supervision of José Ferreirós, and holds a MA in Logic and Philosophy of Science from the Universidad de Granada and a MA in Archaeology from the Universidad de Sevilla. His thesis is (provisionally) entitled «The Genesis of Geometric Knowledge: epistemology, cognitive sciences and history of geometry».

Address: Departamento de Filosofía y Lógica y Filosofía de la Ciencia. Facultad de Filosofía, Universidad de Sevilla. C/ Camilo José Cela s/n, 41018, Sevilla. Email: manueljgarciaperez@gmail.com 